\title{
The role of autophagy in the treatment of pancreatic cancer with gemcitabine and ionizing radiation
}

\author{
HIDEYO MUKUBOU ${ }^{1}$, TOSHIAKI TSUJIMURA ${ }^{1}$, RYOHEI SASAKI $^{2}$ and YONSON KU ${ }^{1}$ \\ Departments of ${ }^{1}$ Surgery, Division of Hepato-Biliary-Pancreatic Surgery, and ${ }^{2}$ Radiation Oncology, \\ Kobe University, 7-5-2 Kusunoki-cho, Chuo-ku, Kobe 650-0017, Japan
}

Received April 7, 2010; Accepted May 25, 2010

DOI: 10.3892/ijo_00000732

\begin{abstract}
Autophagy has recently emerged as a significant mechanism in cancer treatment. Although gemcitabine and/or ionizing radiation are important modalities in the treatment of pancreatic cancer, the contribution of autophagy in such treatment has not been fully elucidated. This study investigated the role of autophagy in the treatment of pancreatic cancer with gemcitabine and ionizing radiation. To evaluate the effect of gemcitabine and/or ionizing radiation on autophagy, several human pancreatic cancer cell lines were used. The treatment of pancreatic cancer cell cultures in vitro and in vivo with gemcitabine and ionizing radiation resulted in synergistic cytotoxicity. After treatment with gemcitabine, the autophagy-related protein light chain 3-II (LC3-II) was upregulated. When gemcitabine was combined with ionizing radiation treatment, LC3-II upregulation was enhanced. In addition, electron microscopy of pancreatic cancer cells treated with gemcitabine and/or ionizing radiation detected the induction of autophagy. The blockage of autophagy by 3methyladenine indicated that autophagy contributed to cell death after gemcitabine treatment and enhanced its cytotoxicity. The inhibitory effect and immune reactivity of the autophagy-related proteins LC3 and beclin-1 were the strongest after the combination treatment. In conclusion, these results suggest that autophagy can be activated by gemcitabine and/ or ionizing radiation in the treatment of pancreatic cancer cells and that activated autophagy plays a role in cancer suppression. These findings may have important implications for future therapeutic strategies using gemcitabine and ionizing radiation against pancreatic cancer.
\end{abstract}

\section{Introduction}

Pancreatic cancer (PC) is an intractable tumor due to its aggressive local invasion and early metastasis. Despite the progress in diagnostic imaging, it is still difficult to detect PC at an early stage (1). Only $20-30 \%$ of PC cases can be treated

Correspondence to: Dr Toshiaki Tsujimura, Department of Surgery, Division of Hepato-Biliary-Pancreatic Surgery, Kobe University, 7-5-2 Kusunoki-cho, Chuo-ku, Kobe 650-0017, Japan E-mail: toshi226@gmail.com

Key words: autophagy, pancreatic cancer, gemcitabine, radiation by surgery, and the prognosis after a single surgical treatment is poor, even after a curative resection. Therefore, chemotherapy (gemcitabine; GEM) and radiotherapy (ionizing radiation; IR) are important modalities for the treatment of PC (2-6).

Autophagy, a type of non-apoptotic cell death, is characterized by sequestration of bulk cytoplasm and organelles in autophagic vesicles (also named autophagosomes) that are fused with lysosomes to generate autolysosomes and are degraded by the cells' own lysosomal system (7). Autophagy plays essential roles in cell survival, growth and development, and tumorigenesis (8-10). Such cell death has profound effects on cancer progression or suppression.

The present study evaluated the contribution of GEM and/or IR-induced autophagy in the treatment of PC by using cells from various human PC cell lines. Clarifying the role of autophagy in the treatment of PC may have important implications for future strategies involving GEM and/or IR.

\section{Materials and methods}

Pancreatic carcinoma cell lines and cell culture. Human PC cell lines PANC-1, AsPC1, and MIAPaCa-2 were obtained from the American Type Culture Collection (Rockville, MD, USA). PANC-1 and AsPC1 were maintained in RPMI-1640 medium (Sigma-Aldrich, St. Louis, MO, USA), and MIAPaCa-2 was maintained in Dulbecco's modified Eagle's medium (Gibco Invitrogen, Carlsbad, CA, USA). Both media were supplemented with $10 \%$ fetal bovine serum (SigmaAldrich), $100 \mathrm{U} / \mathrm{ml}$ penicillin, and $100 \mu \mathrm{g} / \mathrm{ml}$ streptomycin (Invitrogen, Carlsbad, CA, USA). Specimens were kept at $37^{\circ} \mathrm{C}$ in a humidified $5 \% \mathrm{CO}_{2}$ incubator.

Antibodies and reagents. The following antibodies were used in this study: anti-light chain 3 (LC3)-I/II) polyclonal antibody (Medical and Biological Laboratories Ltd., Nagoya, Japan), and beclin-1 and $\beta$-actin polyclonal antibodies (Santa Cruz Biotechnology, Santa Cruz, CA, USA). Other chemical compounds, including 3-methyladenine (3-MA), were purchased from Sigma Chemicals.

Cytotoxicity assay, long-term survival assay, and cell growth curve. The cytotoxic effects of GEM were evaluated using a 3-(4,5-dimethylthiazol-2-yl)-2,5-diphenyl tetrazolium bromide (MTT; Nacalai, Kyoto, Japan) assay. Cells were treated with 
the indicated concentrations of GEM (a gift from Eli Lilly, Japan) or GEM combined with 3-MA (1 mM for $1 \mathrm{~h}$ ) at $37^{\circ} \mathrm{C}$ for $1,3,5$, and 7 days. Initially, moderately cytotoxic doses of 1-200 nM of GEM were evaluated using PANC-1, MIAPaCa-2, and AsPC1 cells to determine the appropriate dose of GEM. Various incubation times (1, 3, 5 and 7 days) were also examined. Absorbance at $570 \mathrm{~nm}$ was measured using a microplate reader (Bio-Rad, Tokyo, Japan). A colonyforming assay was performed to assess the effect of GEM and/or IR. To evaluate GEM as a potential radiosensitizer, PANC-1 and MIAPaCa-2 cells were treated with GEM at concentrations of $0 \mathrm{nM}, \mathrm{IC}_{50} \mathrm{nM}$, and double $\mathrm{IC}_{50} \mathrm{nM}$ for $24 \mathrm{~h}$ (using the MTT assay data for the $\mathrm{IC}_{50}$ dose) and were then exposed to $0,2,4,6$, or $8 \mathrm{~Gy}(2.5 \mathrm{~Gy} / \mathrm{min})$ under the medium condition containing the above doses of GEM. The treated cells were plated onto a new $10-\mathrm{cm}$ tissue culture dish with flesh medium not containing GEM, and the cells were incubated until the cell population completed forming colonies. After fixing and staining, any colonies consisting of more than 50 cells were counted. The surviving fractions were described using a linear-quadratic equation. Decreasing graph lines proved that the sensitization had occurred $(11,12)$. Cell growth curves were graphed for PANC-1 and MIAPaCa-2 cells treated with 3 -MA. PANC-1 (1x105/well) and MIAPaCa- 2 cells $\left(1 \times 10^{5} /\right.$ well $)$ were seeded in a $10-\mathrm{cm}$ dish, precultured for $24 \mathrm{~h}$ in medium $+10 \%$ FBS. The cells were then treated with 3-MA at concentrations of $0 \mu \mathrm{M}, 100 \mu \mathrm{M} \mathrm{x}$ $1 \mathrm{~h}, 1 \mathrm{mM} \times 1 \mathrm{~h}$, and $10 \mathrm{mM} \times 1 \mathrm{~h}$ and cultured for 12,24 , 48 , and $72 \mathrm{~h}$.

Western blot analysis. Equal numbers of control or experimental cells (PANC-1 and MIAPaCa-2) treated with $\mathrm{IC}_{50} \mathrm{nM}$ of GEM for $24 \mathrm{~h}$ and/or $5 \mathrm{~Gy}$ of IR $(2.5 \mathrm{~Gy} / \mathrm{min})$ were lysed using a lysis buffer consisting of Tris- $\mathrm{HCl}$ (pH 7.5), $50 \mathrm{mM}$ $\mathrm{NaCl}, 2 \mathrm{mM}$ ethylenediaminetetraacetic acid (EDTA), $1 \%$ Triton X-100, $10 \mathrm{mM}$ sodium pyrophosphate, $1 \mathrm{mM}$ sodium vanadate, $50 \mathrm{mM}$ sodium fluoride, $1.0 \mathrm{~g} / \mathrm{ml}$ leupeptin, $1.0 \mathrm{~g} / \mathrm{ml}$ aprotinin, $1.0 \mathrm{~g} / \mathrm{ml}$ pepstatin, and $1 \mathrm{mM}$ phenylmethylsulfonyl fluoride. Equivalent amounts of total protein $(20 \mu \mathrm{g})$ were separated by $16 \%$ sodium dodecyl sulfate (SDS) polyacrylamide gel electrophoresis and transferred to nitrocellulose membranes (Portran; Schleicher and Schuell, Dassel, Germany). The membranes were blocked in 5\% non-fat milk for $30 \mathrm{~min}$ at room temperature, washed 3 times, and then incubated with anti-LC3-I/II or B-actin antibodies overnight at $4^{\circ} \mathrm{C}$. LC3-II is localized in autophagosome membranes during amino acid starvation-induced autophagy. Therefore, the amount of LC3-II present in the autophagosome membrane can be indicative of the extent of autophagosome formation (13). The primary antibodies were diluted 1:1000 and detected using the appropriate horseradish peroxidase-conjugated secondary antibodies. Immune complexes were detected by chemiluminescence and then by fluorescence using a LAS3000 mini lumino image-analyzer (Fujifilm, Tokyo, Japan). The density of each band was measured numerically using Multi Gauge Version 3.0 (Fujifilm), and the obtained findings were graphed in a bar chart (LC3-II/ß-actin).

Detection of apoptosis. The induction of apoptosis was evaluated by a DNA fragmentation assay using the APO-Direct ${ }^{\mathrm{TM}}$
Assay Staining kit (BD Bioscience Pharmingen, San Diego, CA, USA). The APO-Direct assay is a single-step method for labeling DNA breaks with fluorescein isothiocyanatedeoxyuridine triphosphate (FITC-dUTP) followed by analysis using flow cytometry (FACS Calibur; Becton-Dickinson, Franklin Lakes, NJ, USA). This method is often used to detect fragmented DNA, and it uses a reaction catalyzed by exogenous TdT, often referred to as 'end-labeling' or 'terminal deoxynucleotidyl transferase dUTP nick and labeling' (TUNEL). We used PANC-1 cells and MIAPaCa-2 cells treated with $\mathrm{IC}_{50} \mathrm{nM}$ of GEM for $24 \mathrm{~h}$ and/or $5 \mathrm{~Gy}$ of IR (2.5 Gy/min) combined with or without 3-MA (1 mM for $1 \mathrm{~h}$ ). Cultured cells were harvested by trypsinization and washed with phosphate buffered saline (PBS). Washed cells were fixed with $1 \%$ paraformaldehyde for $15 \mathrm{~min}$ and then with $70 \%$ ethanol overnight at $-20^{\circ} \mathrm{C}$. Fixed cells were combined with a DNA labeling solution containing conjugated FITC for $30 \mathrm{~min}$. The stained cells were resuspended in PBS, and flow cytometry data were analyzed with CellQuest software (Becton-Dickinson). We measured the cells that were apoptotic as a percentage of the total number of cells using the CellQuest software package.

Electron microscopic analysis. The control and the GEMand/or IR-treated cells were fixed with $2 \%$ glutaraldehyde, $4 \%$ paraformaldehyde, and $1 \%$ tannic acid in $0.1 \mathrm{~mol} / 1$ cacodylate buffer ( $\mathrm{pH} 7.4$ ) for $24 \mathrm{~h}$. After thiocarbohydrazideosmium staining, the cells were dehydrated in a graded ethanol series and then immersed serially in 1:1 hexamethyldisilazane and absolute ethanol. Thin-cut sections and the gels were coated with $500-\AA$ of gold in a JEOL Vacuum sputter coater; they were then viewed using a T300 electron microscope with a scanning attachment (JEOL, Tokyo, Japan). By using the images obtained by electron microscopy, we identified cells having 10 or more autophagic vacuoles in the cell cytoplasm as undergoing 'cell-induced autophagy'. Ten slices at the same magnification were selected at random, and the number of cells in each group was graphed.

In vivo tumor growth assay. Male BALB/c nude mice aged 4-6 weeks (CLEA, Tokyo, Japan) were used. Equal numbers $\left(2 \times 10^{6}\right)$ of MIAPaCa- 2 cells were injected subcutaneously into the hind limbs of the mice to form a tumor xenograft; GEM was then injected intraperitoneally. For tumor irradiation, the mice were immobilized in a customized harness that exposed the hind leg while shielding the remainder of the body with $100 \mathrm{~cm}^{2}$ of lead. Each mouse was then exposed to a single dose of $5 \mathrm{~Gy}(2.5 \mathrm{~Gy} / \mathrm{min})$. The mice were then randomized into 4 groups: sham, GEM alone (300 mg/kg/week), IR alone, and GEM combined with IR (GEM + IR). Each group consisted of 6 mice. The tumor volumes were measured, and the volume was calculated twice weekly using the formula $\mathrm{L} \mathrm{x} \mathrm{W}^{2} / 2$, where $\mathrm{L}$ is the longest diameter and $\mathrm{W}$ is the shortest diameter of the tumor, to assess the antitumor effect of GEM or IR. The 6 mice in each group were maintained in order to observe survival times, and Kaplan-Meier survival curves were constructed.

Immunohistochemical analysis. The excised tumors were fixed in $10 \%$ formalin and then embedded in paraffin. Sections $(4 \mu \mathrm{m}$ 
(A)

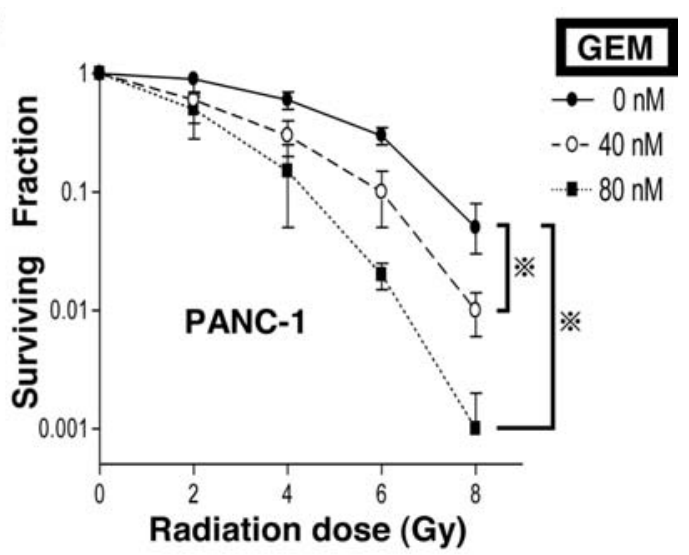

(B)

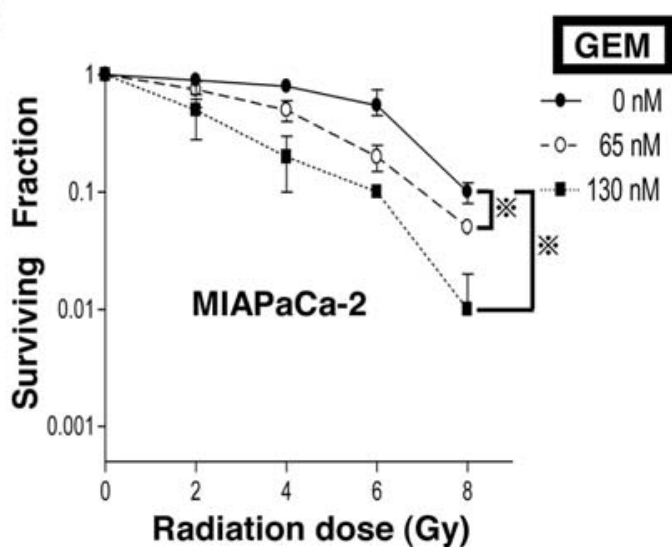

Figure 1. Synergistic effects of GEM and ionizing radiation (IR). (A) PANC-1: (•) IR alone; (o) IR + GEM 40 nM; (•) IR + GEM 80 nM. (B) MIAPaCa-2: (•) IR alone; (०) IR + GEM $65 \mathrm{nM}$; (•) IR + GEM $130 \mathrm{nM}$. IR dose was 0 , 2, 4, 6 and $8 \mathrm{~Gy}$. Error bars were calculated from 12 independent experiments. $\mathrm{P}<0.01$ were considered to be statistically significant; GEM-free line vs. either the GEM $\left(\mathrm{IC}_{50}\right)$ line $(*)$ or the GEM (double $\left.\mathrm{IC}_{50}\right)$ line $(*)$.

Table I. In vitro cytotoxicity of gemcitabine $\left(\mathrm{IC}_{50}\right)$ against pancreatic cancer cell lines by MTT assay.

\begin{tabular}{cccc}
\hline & \multicolumn{3}{c}{$\mathrm{IC}_{50}(\mathrm{nM})$} \\
\cline { 2 - 4 } $\begin{array}{c}\text { Incubation time } \\
\text { (days) }\end{array}$ & PANC-1 & MIAPaCa-2 & AsPC1 \\
\hline 1 & $40 \pm 5.2$ & $65 \pm 4.4$ & $38 \pm 3.2$ \\
3 & $31 \pm 4.3$ & $58 \pm 4.1$ & $35 \pm 2.1$ \\
5 & $20 \pm 5.5$ & $35 \pm 4.1$ & $27 \pm 2.9$ \\
7 & $13 \pm 3.3$ & $28 \pm 4.4$ & $24 \pm 5.2$ \\
\hline
\end{tabular}

$\mathrm{IC}_{50}$ is shown as $\mathrm{nM}$ and the mean $\pm \mathrm{SEM}$ of examined samples.

thick) were stained with anti-LC3 or anti-beclin-1 rabbit polyclonal antiserum (1:50) and incubated with blocking buffer for $40 \mathrm{~min}$ at room temperature, followed by incubation with the primary antibodies overnight at $4^{\circ} \mathrm{C}$. After incubation with the appropriate concentrations of secondary antibody, antibodies were detected in the sections using the ImmunoCruz System (Santa Cruz Biotechnology). The sections were then counterstained with hematoxylin. By using images obtained

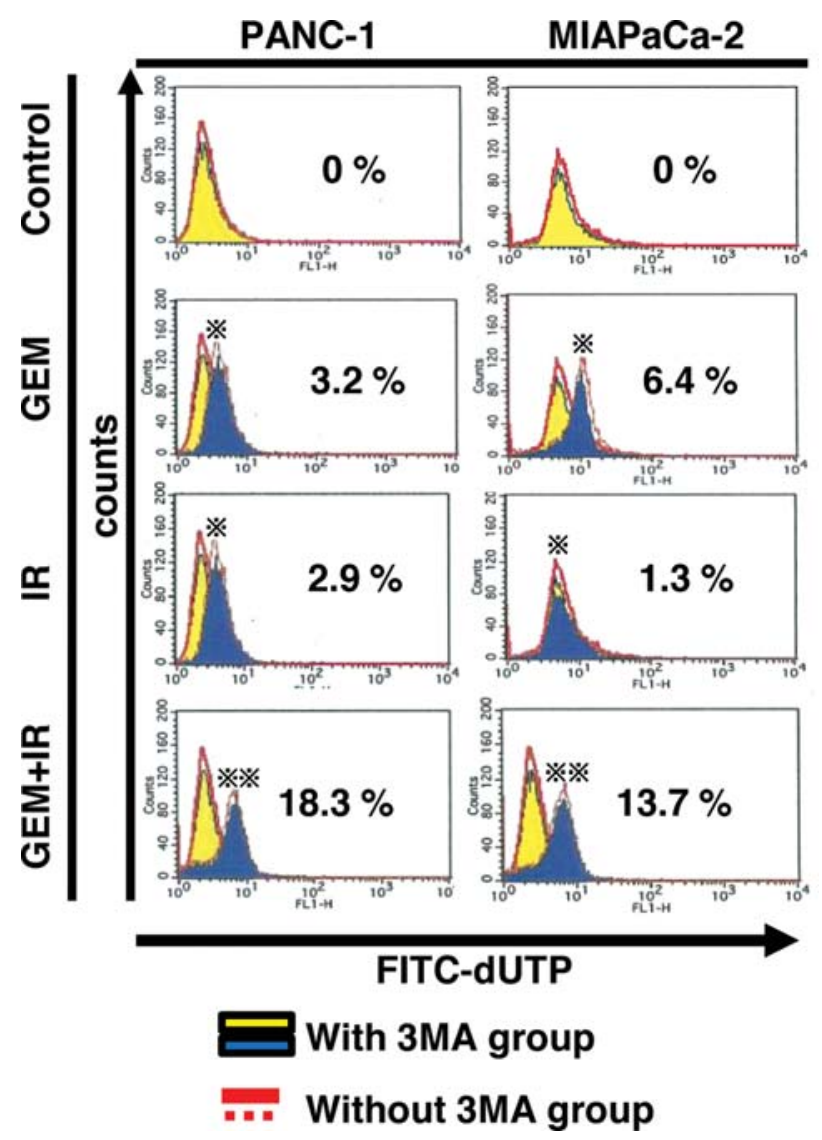

Figure 2. Detection of apoptosis by GEM, IR, and GEM + IR combined with or without 3-methyladenine (3-MA: $1 \mathrm{mM} \times 1 \mathrm{~h}$ ) in PC cells. The $\mathrm{IC}_{50}$ concentration of GEM was administered for $24 \mathrm{~h}$, and $5 \mathrm{~Gy}$ of IR was administered at the rate of $2.5 \mathrm{~Gy} / \mathrm{min}$ for $2 \mathrm{~min}$. $\mathrm{P}<0.01$ were considered to be statistically significant; control group vs. either the GEM group (*) or IR group (*), and the GEM group vs. the GEM + IR group (*).

by light microscopy, we counted the number of cells stained for either LC3 or beclin-1 per 100 cells in 10 slices that had been selected at random. The number of cells for each group was then graphed.

Statistical analysis. Statistical analysis was performed using the GraphPad Prism software program (GraphPad Software, La Jolla, CA, USA) and the StatView Version 5.0 statistical software package (Abacus Concepts, Berkeley, CA, USA). Comparisons were made using two-tailed Student's t-test, and a significant difference was defined as $\mathrm{P}<0.05$. Data are shown as the mean \pm standard deviation (SD). The $\chi^{2}$ test for two-by-two tables was used to compare categorical data. The log-rank test was used to assess the statistical significance of the in vitro and in vivo analyses. The overall survival rates were calculated using the Kaplan-Meier methods. Statistical significance of differences in survival rates were examined using log-rank test for univariate analysis.

\section{Results}

GEM-induced cytotoxicity and the synergistic effect of IR and cell growth curves. We examined the cytotoxic effects of GEM at various incubation times $(1,3,5$ and 7 days) on the 

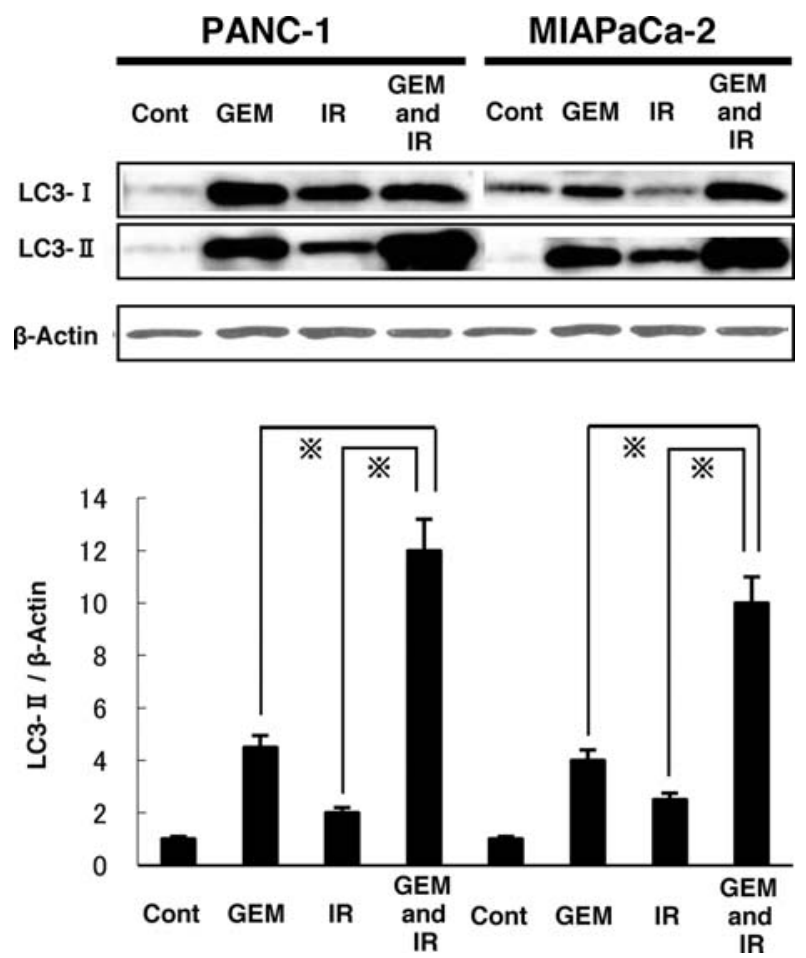

Figure 3. Western blot analysis of LC3 protein in PANC-1 and MIAPaCa-2 cells. PC cells were treated with GEM ( $\mathrm{IC}_{50} \mathrm{nM}$ for $24 \mathrm{~h}$ ) with or without IR (a total of $5 \mathrm{~Gy}$ exposed at a rate of $2.5 \mathrm{~Gy} / \mathrm{min}$ ). The bar chart presents the findings from the quantitative measurement of band densities (LC3-II/ $/$-actin). $\beta$-actin was used as loading control. ${ }^{*} \mathrm{P}<0.01$ were considered to be statistically significant in comparing the GEM + IR group vs. either the GEM group or the IR group.

(A)

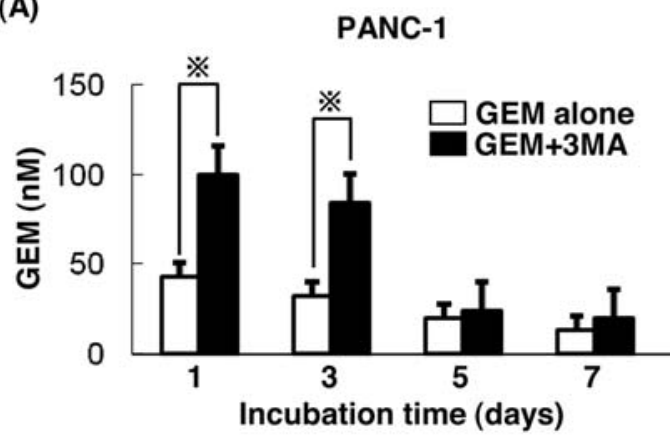

(B)

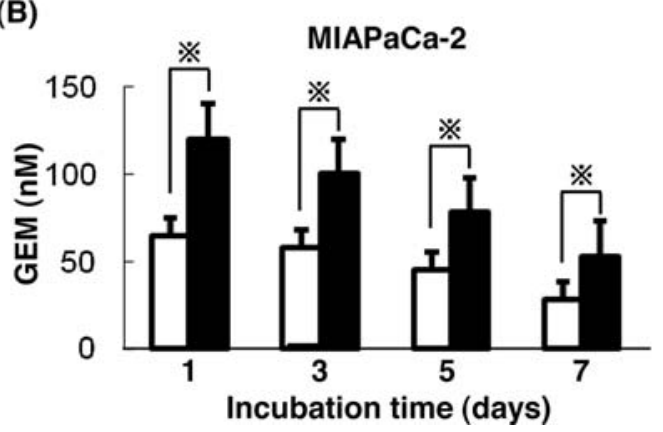

Figure 5. Effect of the autophagy inhibitor 3-methyladenine (3-MA) in PANC-1 (A) and MIAPaCa-2 cells (B) in vitro. 3-MA inhibits the formation of autophagosomes. Toxicity was detected using the MTT assay. GEM concentration shows the mean concentration of $\mathrm{IC}_{50} \cdot{ }^{*} \mathrm{P}<0.05$ were considered to be statistically significant in comparing GEM alone vs. GEM + 3-MA.
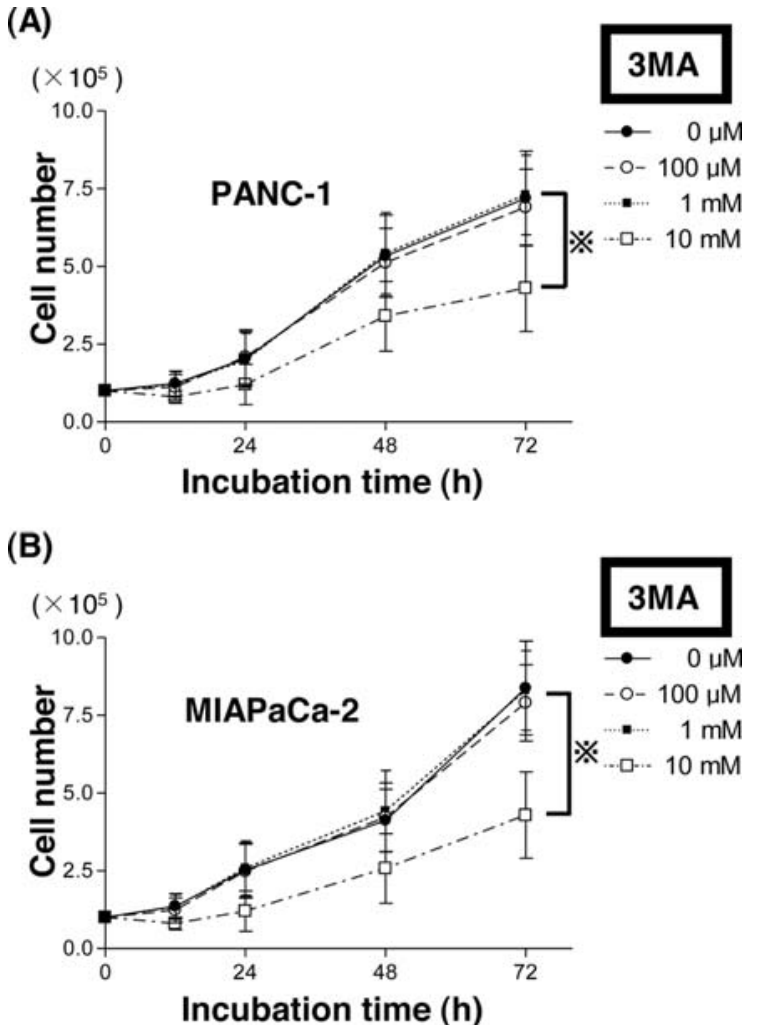

Figure 4. Cell growth curves of PANC-1 (A) and MIAPaCa-2 cells (B) following treatment with 3-MA. PANC-1 and MIAPaCa-2 cells were cultured for $12,24,48$ and $72 \mathrm{~h}$ with 3-MA at concentrations of $100 \mu \mathrm{M}, 1 \mathrm{mM}$ and $10 \mathrm{mM} . \mathrm{P}<0.01$ were considered to be statistically significant; $3-\mathrm{MA}(0 \mu \mathrm{M} /$ $100 \mu \mathrm{M} / 1 \mathrm{mM})$ line and vs. the 3-MA (10 mM) line (*).

PANC-1, AsPC1, and MIAPaCa-2 PC cell lines using an MTT assay. After a 24-h incubation period, the $3 \mathrm{PC}$ cell lines showed similar levels of GEM-induced cytotoxicity ( $\mathrm{IC}_{50}$ concen-trations $38-65 \mathrm{nM}$ ). This trend continued as incubation periods were lengthened, with enhancement of GEM-induced cyto-toxicity occurring in a time-dependent manner (Table I). We performed a colony-forming assay to determine the radio-sensitizing potential of GEM. The surviving fractions were described using a linear-quadratic equation. Decreasing graph lines proved that sensitization was demonstrated. In comparison to the GEM-free group, both the GEM $\left(\mathrm{IC}_{50}\right)$ group line $(\mathrm{P}<0.01)$ and the GEM (double $\left.\mathrm{IC}_{50}\right)$ group line $(\mathrm{P}<0.01)$ decreased significantly in PANC-1 and MIAPaCa- 2 cells. Therefore, synergistic effects were observed in the colony-forming assay when GEM was combined with IR (Fig. 1).

Induction of apoptosis by GEM and/or IR with or without 3-MA and determination of the GEM-induced apoptotic index. The induction of apoptosis was also assessed in the PANC-1 and MIAPaCa-2 PC cell lines (Fig. 2). As the graph shows, the inhibition of autophagy by 3-MA did not influence the proportion of apoptotic cells in these groups. A single treatment with GEM or IR increased the apoptotic cell population in PANC-1 (GEM, 3.2\% and IR, 2.9\%) and MIAPaCa-2 cells (GEM, 6.4\% and IR, 1.3\%). Combined GEM + IR treatment increased the proportion of apoptotic cells significantly more than GEM or IR treatment alone in both PANC-1 (18.3\%, $\mathrm{P}<0.01)$ and MIAPaCa-2 cells $(13.7 \%, \mathrm{P}<0.01)$. 
(A)

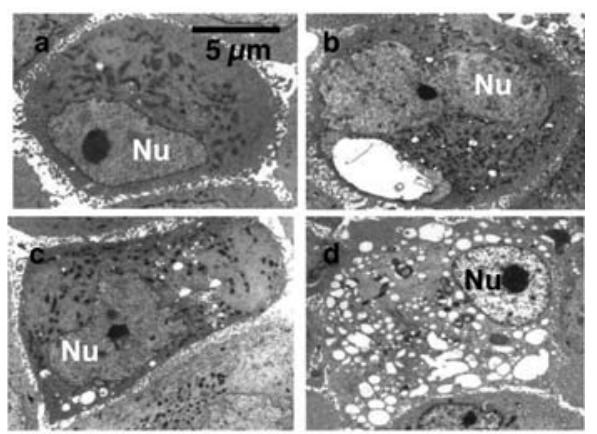

(B)

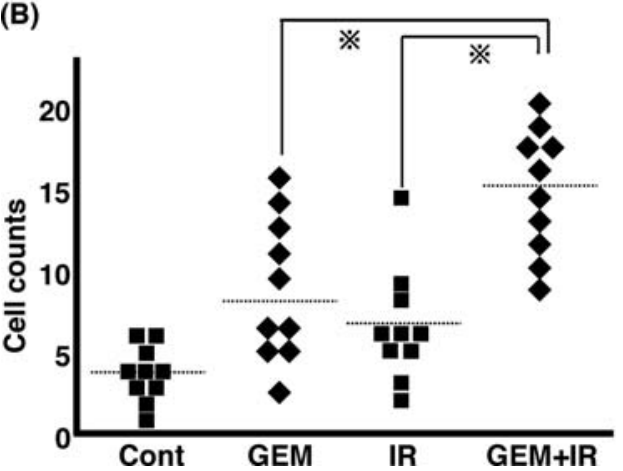

Figure 6. (A) Induction of autophagy evaluated by electron microscopy Electron microscopy images show autophagic vacuoles in PANC-1 cells. (A) Control (a), GEM (b; $\mathrm{IC}_{50} \mathrm{nM}$ for $24 \mathrm{~h}$ ), IR (c; 5 Gy exposed at $2.5 \mathrm{~Gy} / \mathrm{min}$ ), and GEM + IR (d; GEM; $\mathrm{IC}_{50} \mathrm{nM}$ for $24 \mathrm{~h}$ and IR 5 Gy exposed at $\left.2.5 \mathrm{~Gy} / \mathrm{min}\right)$. (B) Frequencies of cells with autophagy induced by GEM, IR, and GEM + IR in PANC-1 cells. The graph shows the cells counts of those with 10 or more autophagic vacuoles in the cytoplasm for each group. ${ }^{*} \mathrm{P}<0.01$.
Detection of autophagy in the treatment with GEM and/or $I R$. The expression of LC3-I (18 kDa) and LC3-II (16 kDa) was examined in PANC-1 and MIAPaCa- 2 cells treated with GEM and/or IR (Fig. 3). In comparison to the cells treated with GEM or IR alone, combined treatment in both PANC-1 and MIAPaCa-2 cells induced significantly higher levels of LC3-II ( $\mathrm{P}<0.01$; LC3-II/ß-actin).

Inhibition of autophagy by the specific inhibitor 3-MA. As shown in Fig. 4, cell growth curves showed that PANC-1 and MIAPaCa-2 PC cell lines had reduced tumor growth following treatment with 3-MA at a concentration of $10 \mathrm{mM} \times 1 \mathrm{~h}$ compared to those treated with concentrations of $0 \mu \mathrm{M}$, $100 \mu \mathrm{M} \times 1 \mathrm{~h}$, and $1 \mathrm{mM} \times 1 \mathrm{~h}$. Therefore, in the MTT assay experiments, the 3-MA concentration used was $1 \mathrm{mM} \times 1 \mathrm{~h}$ since 3-MA treatment was not toxic at this concentration. The effect of 3-MA on GEM-induced cytotoxicity was examined in vitro (Fig. 5). In the PANC-1 and MIAPaCa-2 PC cell lines, 3-MA inhibited GEM-induced cytotoxicity following 1, 3, 5 and 7 days of incubation. The 3-MA-treated cells required significantly higher concentrations of GEM to suppress cell proliferation than did the untreated cells $(\mathrm{P}<0.05)$.

Detection of autophagy by electron microscopic analysis. Induction of autophagy was evaluated by electron microscopy. Mitochondrial disruption was observed in autophagic vacuoles in PANC-1 cells treated with the GEM, IR, and the combination of GEM + IR (Fig. 6A). Compared to the cells treated with a single treatment of GEM or IR, the number of
(A)

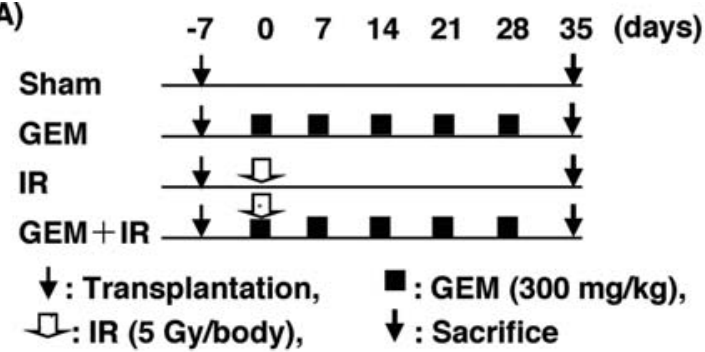

(C)

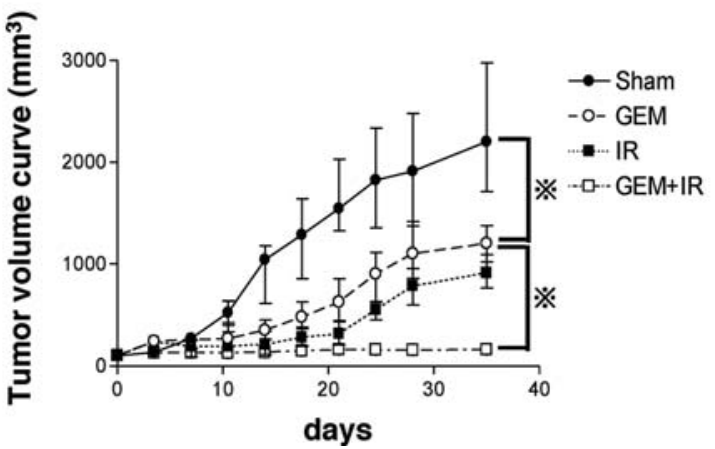

(B)

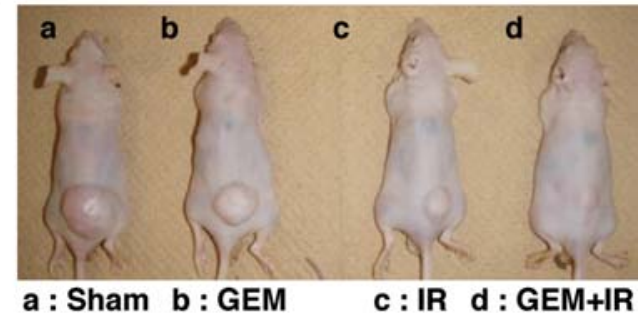

(D)

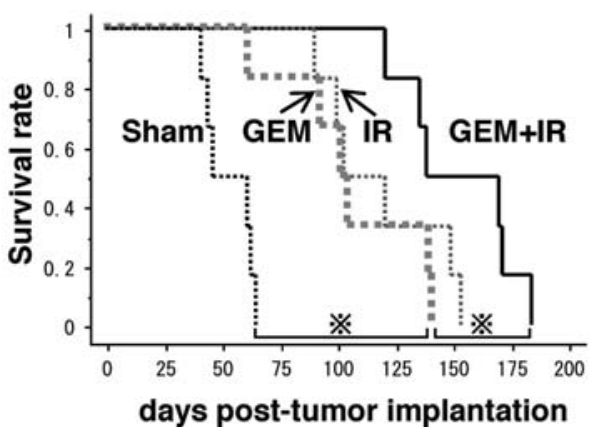

Figure 7. Enhancement of gemcitabine (GEM) antitumor effects by ionizing radiation (IR) on the growth of MIAPaCa-2 human PC xenografts in vivo. Male $\mathrm{BALB} / \mathrm{c}$ nude mice aged 4-6 weeks were randomized into 4 groups: sham, GEM alone, IR alone, and GEM combined with IR (GEM + IR). Each group consisted of 6 mice. According to the protocol (A), the mice were exposed to a single dose of 5 Gy and $300 \mathrm{mg} / \mathrm{kg} / \mathrm{week}$; GEM was administered intraperitoneally. (B) The general appearance of representative mice bearing MIAPaCa-2 xenografts after 35 days. (C) The tumor volume curves on the growth of MIAPaCa-2 xenografts. Individual mice were monitored for tumor growth over a period of 35 days. The volume for individual tumors was calculated and each curve represents the mean tumor volume calculated for each group $(n=6)$. Standard errors of the mean are represented by error bars. (D) The survival curve was plotted according to the methods of Kaplan-Meier $(n=6){ }^{*} \mathrm{P}<0.05$ were considered to be statistically significant for sham vs. either the GEM group or the IR group. ${ }^{* *} \mathrm{P}<0.05$ were considered to be statistically significant for GEM + IR vs. either GEM or IR alone. 
(A)
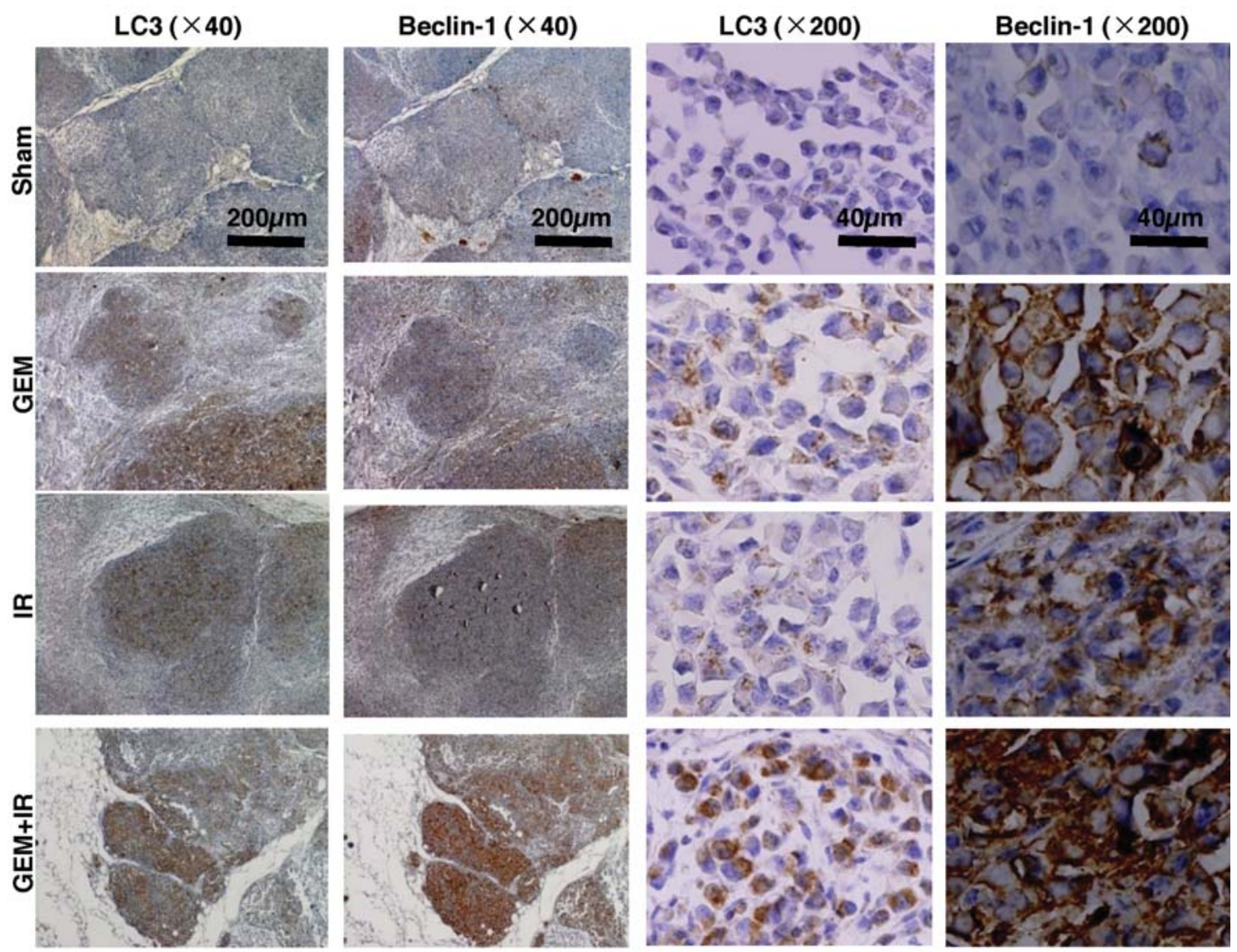

(B)
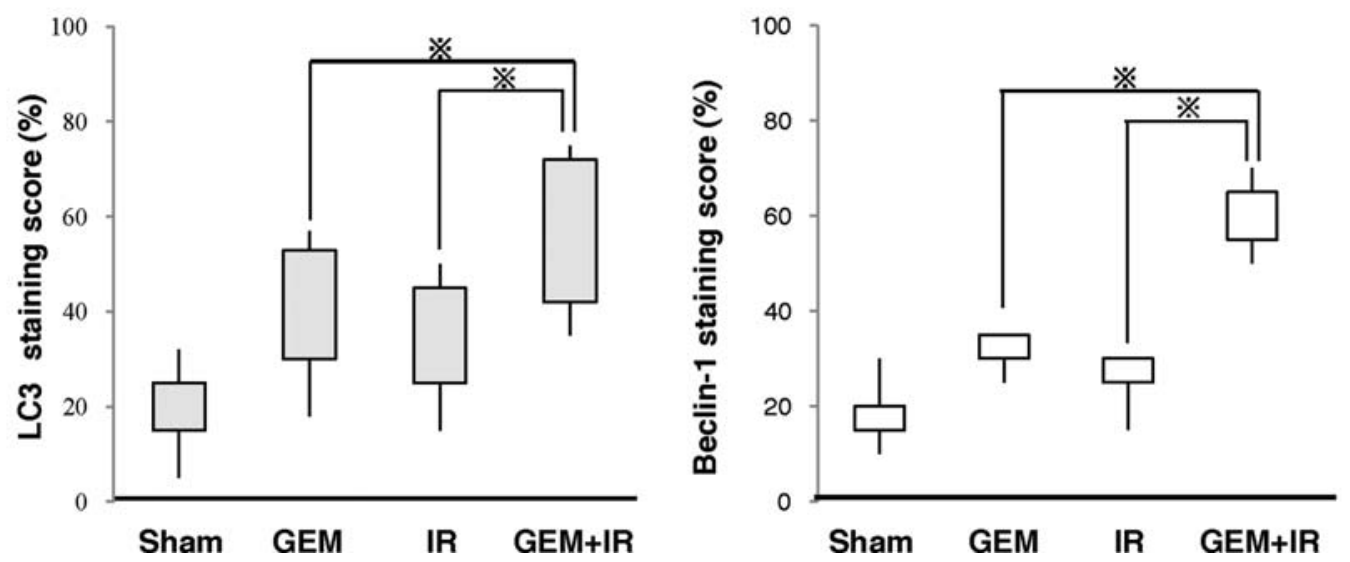

Figure 8. Primary tumors (sham, GEM-treated tumor, IR-treated tumor, and GEM + IR-treated tumor) were fixed and then embedded in paraffin. The sections were stained with LC3, beclin-1 antibodies (A). The sections were counterstained with hematoxylin. (B) From the images obtained by light microscopy, we counted the number of cells staining for either LC3 or beclin-1 per 100 cells in 10 slices selected at random. The number of cells for each group is shown in the graph. ${ }^{*} \mathrm{P}<0.01$ were considered to be statistically significant in comparing the GEM + IR group vs. either the GEM group or the IR group.

autophagic cells was significantly greater when treated in combination with GEM + IR, suggesting that GEM combined with IR induces autophagy synergistically (Fig. 6B).

Growth inhibitory effects of GEM and/or IR in PC xenografts. The effect of IR combined with GEM on tumor growth was further investigated with an in vivo MIAPaCa-2 mouse model.
All animal experiments were performed strictly according to the institutional ethics guidelines. GEM was injected intraperitoneally into the mice, and IR was locally administered to the tumor (Fig. 7A). Representative results from each mouse group are shown in Fig. 7B. Xenograft growth speed was moderately reduced when the mice were treated with either IR or GEM alone. In contrast, the tumor 
size significantly decreased after combined GEM + IR treatment $(\mathrm{P}<0.05$; Fig. 7C). As shown in Fig. 7D, the median survival time of mice was 53 days in the sham group, whereas it was significantly increased to 107,119 , and 153 days in the GEM ( $\mathrm{P}=0.0034)$, IR $(\mathrm{P}=0.0025)$, and GEM + IR $(\mathrm{P}<0.0001)$ groups, respectively. Survival time in the GEM + IR group was prolonged by $289 \%$ when compared with the sham group.

Immunohistochemical analyses of autophagy-related proteins by each treatment. Immunohistochemical analysis of LC3 and beclin-1 was performed using tumors obtained after each treatment (Fig. 8A). In comparison to the cells treated with GEM or IR alone, a synergistic effect was observed when GEM was combined with IR $(\mathrm{P}<0.01)$. Quantitative evaluation by counting positive cells showed that the synergistic effects were induced by the combination, as shown in Fig. 8B.

\section{Discussion}

It is generally agreed that autophagic activity contributes to the development of cancers. However, there is continuing controversy as to whether induced autophagy results in cancer suppression or progression. Kondo et al noted that there is no consensus as to whether autophagy is a cause of cell death or whether autophagy occurs in an effort to avoid cell death (14). Yang et al reported that autophagy has different effects on cell survival depending on the stage of cell death (15). Oginer-Denis et al suggested that suppression of autophagy may contribute to the initial rapid growth of tumors, however, in more advanced stages of cancer, autophagy may be required to provide essential nutrients to the cells in the inner part of solid tumors that do not have direct access to circulation (16). Therefore, some of the recently suggested strategies for cancer treatment include inducing autophagy in early developed cancers while inhibiting autophagy in advanced tumor cells with intact autophagy responses to sensitize the cells to a variety of anti-cancer agents $(10,17)$.

Some studies have shown that activated autophagy is a tumor suppressor. The overexpression of beclin-1, a mammalian autophagy protein, induces autophagy and contributes to cancer suppression in various cancer cells $(8,18,19,20)$. Decreased expression of autophagy proteins may contribute to the development or progression of breast and other human malignancies (19). Studies of beclin-1 specifically, provided genetic evidence that autophagy is a novel mechanism of cellgrowth control and tumor suppression (20). Additionally, Scarlatti et al reported that tamoxifen causes cell death by autophagy in mammary tumor cells and that the inhibition of autophagy by 3-MA decreased cell death (21). In the present study, we examined the contribution of autophagy in PC cells treated with GEM by applying 3-MA. As shown Fig. 5, the 3-MA-use group required a significantly higher concentration of GEM to suppress PC cells compared with the 3-MA-nonuse group. In addition, as shown in Fig. 6, electron microscopy confirmed the presence of autophagy vacuoles, which disrupt mitochondria, within the cytoplasm in PC cells in the presence of GEM, IR and the combination of the two. Taken together, these results clearly indicate that GEM induces autophagy and that induced autophagy acts on cancer suppression.
In contrast, some reports concluded that autophagy acts on cancer progression. Li et al reported that 5FU-induced apoptosis in colon cancer cells can be enhanced by the inhibitor of autophagy (3-MA) and autophagy may act as a self-defense mechanism in 5FU-treated colon cancer cells (22). Sato et al reported that both the autolysosome inhibitors and 3-MA induced marked apoptotic death of all colorectal cancer cells examined, suggesting that autophagy directly contributes to the survival of cancer cells under nutrient starvation (23). In both reports, however, larger doses of 3-MA were applied for longer exposure times compared to those used in our study (Li et al, $5 \mathrm{mM} \times 36 \mathrm{~h}$, and Sato et al, $10 \mathrm{mM}$ x $12 \mathrm{~h}$, vs. our study, $1 \mathrm{mM}$ x $1 \mathrm{~h}$ ). The apoptotic death reported in these studies may be cytotoxicity caused by high concentrations of 3-MA. As shown in Fig. 4, our results indicated that 3-MA concentrations greater than $1 \mathrm{mM} \times 1 \mathrm{~h}$ were cytotoxic to both PANC-1 and MIAPaCa- 2 cells. At a $1 \mathrm{mM}$ concentration, our results showed that 3-MA could inhibit autophagy and decrease the antitumor effect without inducing apoptosis.

Only a few attempts have been made so far to study the phenomena of autophagy and apoptosis induced by the combination of chemotherapy and radiotherapy. IR alone induced autophagy for breast, prostate, and colon cancer (24). Similarly, IR inhibited cell proliferation and increases autophagy activity in various glioblastoma cells (25). We have examined the phenomena of autophagy and apoptosis with the combined treatment of GEM and IR on PC cells. The expression of LC3-II was high in the GEM and IR groups and was even higher in the combination group in comparison to the control group (Fig. 3). Immunohistochemical assessment of the tumor grafts showed that, while the expression of LC3 and beclin-1 was remarkably enhanced in the GEM and IR groups, it was further enhanced in the combination group (Fig. 8). The proportion of apoptotic cells also increased synergistically following treatment with GEM and IR in combination compared to the use of GEM or IR alone (Fig. 2).

In conclusion, autophagy is induced by the administration of GEM and/or IR to PC cells and that induced autophagy acts to suppress cancer. Further studies are needed to analyze the molecular mechanism of autophagy, and may lead to the development of new treatment strategies for the use of GEM and IR for PC.

\section{Acknowledgements}

We are grateful to Dr T. Kanzawa for his invaluable comments on our experiments and helpful discussion. We wish to thank Yoko Suzuki and Chie Fukui for their excellent technical help. This research was supported by a grant-in-aid for scientific research from the Ministry of Education, Culture, Sports, Science and Technology of Japan to T. Tsujimura (C) (20591526).

\section{References}

1. Huguet F, Andre T, Hammel P, et al: Impact of chemoradiotherapy after disease control with chemotherapy in locally advanced pancreatic adenocarcinoma in GERCOR phase II and III studies. J Clin Oncol 25: 326-331, 2007. 
2. Okusaka T, Ito Y, Ueno H, et al: Phase II study of radiotherapy combined with gemcitabine for locally advanced pancreatic cancer. Br J Cancer 91: 673-677, 2004.

3. Crane CH, Varadhachary G, Wolff RA, Pisters PW and Evans DB: The argument for pre-operative chemoradiation for localized, radiographically resectable pancreatic cancer. Best Pract Res Clin Gastroenterol 20: 365-382, 2006.

4. Gordon AT and McMillan TJ: A role for molecular radiobiology in radiotherapy? Clin Oncol 9: 70-78, 1997.

5. Blackstock AW, Bernard SA, Richards F, et al: Phase I trial of twice-weekly gemcitabine and concurrent radiation in patients with advanced pancreatic cancer. J Clin Oncol 17: 2208-2212, 1999.

6. Rejiba S, Wack S, Aprahamian M and Hajri A: K-ras oncogene silencing strategy reduces tumor growth and enhances gemcitabine chemotherapy efficacy for pancreatic cancer treatment. Cancer Sci 98: 1128-1136, 2007.

7. Klionsky DJ and Emr SD: Autophagy as a regulated pathway of cellular degradation. Science 290: 1717-1721, 2000.

8. Levine B and Klionsky DJ: Development by self-digestion: molecular mechanisms and biological functions of autophagy. Dev Cell 6: 463-477, 2004.

9. Klionsky DJ: Autophagy: from phenomenology to molecular understanding in less than a decade. Nat Rev Mol Cell Biol 8: 931-937, 2007

10. Tan ML, Ooi JP, Ismail N, Moad AI and Muhammad TS: Programmed cell death pathways and current antitumor targets. Pharm Res 26: 1547-1560, 2009.

11. Lawrence TS, Davis MA, Hough A and Rehemtulla A: The role of apoptosis in 2'2'-diflouro-2'-deoxycytidine (gemcitabine)mediated radiosensitization. Clin Cancer Res 7: 314-319, 2001.

12. Cao C, Subhawong T, Albert JM, et al: Inhibition of mammalian target of rapamycin or apoptotic pathway induces autophagy and radiosensitizes PTEN null prostate cancer cells. Cancer Res 66: 10040-10047, 2006

13. Kabeya Y, Mizushima N, Ueno T, et al: LC3, a mammalian homologue of yeast Apg8p, is localized in autophagosome membranes after processing. EMBO J 19: 5720-5728, 2000.

14. Kondo Y, Kanzawa T, Sawaya R and Kondo S: The role of autophagy in cancer development and response to therapy. Nat Rev Cancer 5: 726-734, 2005.
15. Yang YP, Liang ZQ, Gao B, Jia YL and Qin ZH: Dynamic effects of autophagy on arsenic trioxide-induced death of human leukemia cell line HL60 cells. Acta Pharmacol Sin 29: 123-134, 2008.

16. Ogier-Denis E and Codogno P: Autophagy: a barrier or an adaptive response to cancer. Biochim Biophys Acta 1603: 113-128, 2003.

17. Karantza-Wadsworth V and White E: Role of autophagy in breast cancer. Autophagy 3: 610-613, 2007.

18. Liang XH, Kleeman LK, Jiang HH, et al: Protection against fatal Sindbis virus encephalitis by beclin, a novel Bcl-2-interacting protein. J Virol 72: 8586-8596, 1998.

19. Liang XH, Jackson S, Seaman M, et al: Induction of autophagy and inhibition of tumorigenesis by beclin 1 . Nature 402: 672-676, 1999.

20. Qu X, Yu J, Bhagat G, et al: Promotion of tumorigenesis by heterozygous disruption of the beclin 1 autophagy gene. J Clin Invest 112: 1809-1820, 2003.

21. Scarlatti F, Bauvy C, Ventruti A, et al: Ceramide-mediated macroautophagy involves inhibition of protein kinase B and upregulation of beclin 1. J Biol Chem 279: 18384-18391, 2004.

22. Li J, Hou N, Faried A, Tsutsumi S, Takeuchi T and Kuwano H: Inhibition of autophagy by $3 \mathrm{MA}$ enhances the effect of 5FUinduced apoptosis in colon cancer cells. Ann Surg Oncol 16: 761-771, 2009.

23. Sato K, Tsuchihara K, Fujii S, et al: Autophagy is activated in colorectal cancer cells and contributes to the tolerance to nutrient deprivation. Cancer Res 67: 9677-9684, 2007.

24. Paglin S, Lee NY, Nakar C, et al: Rapamycin-sensitive pathway regulates mitochondrial membrane potential, autophagy, and survival in irradiated MCF-7 cells. Cancer Res 65: 11061-11070, 2005.

25. Yao KC, Komata T, Kondo Y, Kanzawa T, Kondo S and Germano IM: Molecular response of human glioblastoma multiforme cells to ionizing radiation: cell cycle arrest, modulation of the expression of cyclin-dependent kinase inhibitors, and autophagy. J Neurosurg 98: 378-384, 2003. 